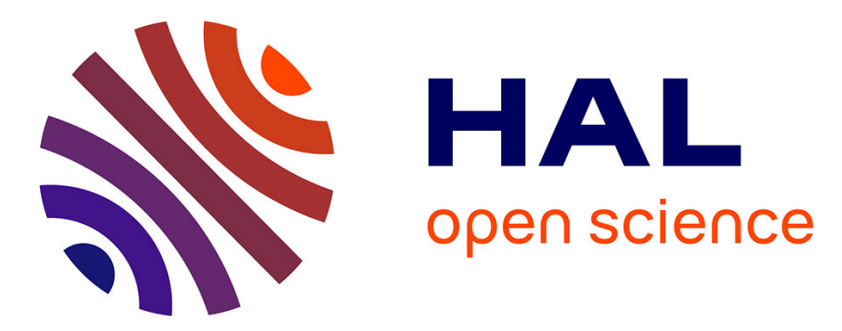

\title{
Impact of the heap shape formation on the local vertical force profile of ensiled granular materials
}

\author{
Agnès Duri-Bechemilh, Frederic Mabille, Thierry Ruiz
}

\section{To cite this version:}

Agnès Duri-Bechemilh, Frederic Mabille, Thierry Ruiz. Impact of the heap shape formation on the local vertical force profile of ensiled granular materials. Powder Technology, 2018, 338, pp.993-1000. 10.1016/j.powtec.2018.06.046 . hal-01604246

\section{HAL Id: hal-01604246 \\ https://hal.science/hal-01604246}

Submitted on 26 May 2020

HAL is a multi-disciplinary open access archive for the deposit and dissemination of scientific research documents, whether they are published or not. The documents may come from teaching and research institutions in France or abroad, or from public or private research centers.
L'archive ouverte pluridisciplinaire HAL, est destinée au dépôt et à la diffusion de documents scientifiques de niveau recherche, publiés ou non, émanant des établissements d'enseignement et de recherche français ou étrangers, des laboratoires publics ou privés.

\section{다(1)(2)}

Distributed under a Creative Commons Attribution - ShareAlikel 4.0 International 


\title{
Impact of the heap shape formation on the local vertical force profile of ensiled granular materials
}

\author{
Duri Agnès*, Mabille Frédéric, Ruiz Thierry, \\ IATE, CIRAD, INRA, Montpellier SupAgro, Université de Montpellier, \\ 34060 Montpellier, France
}

\begin{abstract}
The free surface, which results from the filling of a container by a dry granular material, takes the shape of a heap. This shape is induced by the particle properties like their size, their friction, their restitution coefficient but also by their kinetic energy induced by the filling conditions. The stabilization of the intergranular contact network inside the heap leads to the formation of a specific local stress field. For five wheat-based powders of same origin but with contrasted properties, beds are formed in a $2 \mathrm{D}$ semi-confined cell using a point source. Captures of the bed free surface are realized from pictures taken at different filling states. The local vertical force is measured in the center of the cell at different depths. This work highlights a correlation between the local vertical force profile and the shape of the heap during the filling. Two modes of force distribution can be distinguished and classified according to a parameter of uniformity. These distinctions are linked to the different shapes of the heap, quasi-layering or dune. Our experimental study demonstrates that the history of the powder filling directly impacts on the resulting local force profile.

Keywords: Ensiled granular medium; Vertical force profile; Heap free surface; Wheat-based powders.
\end{abstract}

\footnotetext{
* Corresponding author

Email addresses: agnes.duri@supagro.inra.fr(), Tel.: +33499612060()

$U R L:$ ww. http://umr-iate.cirad.fr/ ()
}

Preprint submitted to Powder Technology 


\section{Introduction}

During a container filling by a dry granular material (e.g. storage in a silo, mixer container, packing), the powder flow interacts with the side walls and the bottom. It leads to the formation of a semi-confined free surface in the 'feeding zone' and particle jamming under the free surface [1]. The resultant free surface takes the shape of a heap and the surface raises upward the container with the powder filling. The stabilization of the intergranular force network inside the heap leads to the formation of a local stress field $[2,3]$. The knowledge of the local stress field is important to evaluate the mechanical stability of storages [4] or the grain re-mobilization under the influence of a shear (mixing, flowing) or a compression (packing, tapping, compaction) stress [5]. This work concerns the experimental identification of relationships between the static mechanical state within an ensiled granular bed and its free surface formation during filling. Different wheat-based powders of same origin but with contrasted properties have been considered in order to elucidate a potential relation between the structure of the free surface, which results from the local granular arrangements, and the local distribution of the vertical force.

\section{Materials and methods}

\subsection{Materials}

Five different wheat powders of $1.47 \mathrm{~g} . \mathrm{cm}^{-3}$ were selected. The main raw material was durum wheat semolina of industrial quality (Panzani group, France). The fine fraction of durum wheat semolina was collected by sieving under a metallic sieve of $315 \mu \mathrm{m}$ mesh. To investigate larger particle size, agglomerated grains of durum wheat semolina, called couscous grains, were selected. Fine size couscous grains and medium size couscous grains came from an industrial production (Zakia, France). The large size couscous grains were collected by sieving the medium size couscous grains over a metallic sieve of $1250 \mu \mathrm{m}$ mesh.

Particle diameters were characterized using laser granulometry (Mastersizer 2000, Malvern, England). The median diameter, $d_{50}$ (i.e. the volume-equivalent 
diameter for which the number of particles is respectively inferior to 50\%), and the size width, $d_{90}-d_{10}$ (where $d_{10}$ and $d_{90}$ are the volume-equivalent diameters for which the number of particles is respectively inferior to $10 \%$ and $90 \%$ ), are given in Table 1. We observe a range of median diameters with a factor of five between the smallest and the largest particles and a large size distribution of widths with lower values for sieved powders (i.e. fine semolina and large couscous).

The apparent friction coefficient, $\mu$, of wheat powders has been measured with a FT4 powder rheometer (Freeman Technology Ltd., Worcestershire) and the angle of repose, $\theta$, was deduced from pictures of the heap obtained by pouring $60 \mathrm{~g}$ of powder over a plane surface (without wall effect). The measurements have been repeated five times and the mean values are reported in Table 1. The apparent friction coefficient and the angle of repose decrease with the median diameter, but the last parameter allows to discriminate semolina and couscous family. In comparison with couscous, semolinas are characterized by a smaller size and frictional properties more important.

The large variability of the particle surface did not allow us to measure the restitution coefficient. However, we qualitatively observed that the rebound properties are contrasted between both families. Couscous grains have a rebound capacity much more important than the one of semolinas.

Table 1: Characteristics of the granular media.

\begin{tabular}{lllll}
\hline Name & $\begin{array}{l}d_{50} \\
(\mathrm{~mm})\end{array}$ & $\begin{array}{l}d_{90}-d_{10} \\
(\mathrm{~mm})\end{array}$ & $\mu(/)$ & $\theta(\mathrm{deg})$ \\
\hline Fine semolina & 0.210 & 0.321 & 0.578 & 33.3 \\
Semolina & 0.298 & 0.434 & 0.415 & 31.5 \\
Fine couscous & 0.676 & 0.645 & 0.390 & 12.1 \\
Medium couscous & 0.953 & 0.517 & 0.291 & 9.9 \\
Large couscous & 1.095 & 0.406 & 0.307 & 7.6 \\
\hline
\end{tabular}




\subsection{Methods}

The local weight profile was defined as the vertical contribution of the force network acting above the surface of a probe immersed in the granular bed. This weight was measured using an original device developed by Mandato et al. [6] and composed of an open glass cell container $(160 \mathrm{~mm}$ height, $100 \mathrm{~mm}$ length, $51 \mathrm{~mm}$ width), a flat and rectangular steel probe (0.97 mm thickness, $44.8 \mathrm{~mm}$ length, $19.7 \mathrm{~mm}$ width) which was screwed on a rod linked to a load cell of a texture analyzer (TAXT Plus, Table Micro System) and used as a force sensor (Fig. 1a-b). The probe was first placed inside the cell container at $0.2 \mathrm{~mm}$ from the front side, in the middle $(x=0 \mathrm{~mm})$ and at the desired distance from the bottom of the cell (Fig. 1c). The probe position was defined according to the depth $(z)$ in the powder bed, from the powder surface $(z=0 \mathrm{~mm})$ to the bottom of the cell. The powder was sprinkled inside the glass cell container with a funnel until to reach a bed height $H$ of $140 \mathrm{~mm}$. We ensured that the granular flow depends on the outlet diameter [7]. Force measurements were performed at each probe position $z$ during $2 \mathrm{~s}$. For a same position, mean values and standard deviations were determined at least from triplicates.

The pictures of the powder bed formation in the cell were obtained with a Nikon D4 camera equipped with a macro lens of $105 \mathrm{~mm}$. The converted images were then treated by Image $\mathrm{J}(\mathrm{R}$ software to determine the free surface of the bed as a function of the depth $z$.

\section{Results and discussion}

\subsection{Bed formation}

For each selected powder, the bed formation has been studied by analyzing their final compactness and their free surface evolution in 2D configuration. The compactness, $\Phi$, has been calculated from the mass and volume of the powder bed (see Table 2$)$. For couscous, its value is approximatively constant $(\approx 0.576)$ and in comparison with semolina, it is about higher for $30 \%$. 
Table 2: Characteristics of the bed and the heap formation.

\begin{tabular}{llll}
\hline Name & $\phi(/)$ & $\bar{f}(\mathrm{~mm})$ & $\bar{\alpha}(\mathrm{deg})$ \\
\hline Fine semolina & 0.416 & 41.17 & 37.6 \\
Semolina & 0.446 & 31.49 & 31.0 \\
Fine couscous & 0.578 & 16.70 & 23.7 \\
Medium couscous & 0.573 & 16.34 & 24.2 \\
Large couscous & 0.578 & 18.33 & 25.2 \\
\hline
\end{tabular}

From pictures, the shape of the free surface was captured as numerical data and then plotted at different steps of the filling (different depths $z$ ). Examples of pictures and captured free surfaces are given in Fig. 2 for semolina and large couscous.

Whatever the wheat-powder and the depth, the shape of the free surface of the heap is globally the same. As shown in Fig. 3b, this shape is not an ideal prism because the top and the base of the heap are rounded off. We observe that the shape of the heap depends on the properties of the powder (Fig. 3a) but also on the depth (Fig. 2). In order to compare all these shapes, we have considered two representative criteria of these curves, the deflection, $f$, and the apparent angle of heap, $\alpha$, deduced from a Gaussian fit of the free surface data (Fig. 3b). The equation used to represent the shape of the free surface is described with a good agreement by :

$$
p(x, z)=z+f(z) \exp ^{-\frac{x^{2}}{2 \omega(z)^{2}}}
$$

$x$ is the horizontal coordinate, $z$ is the depth, $p(x, z)$ is the coordinates of the free surface at each depth, $f(z)$ is the deflection of the heap at each depth, and $2 \omega(z)=\omega_{1}(z) / \sqrt{\ln (4)}$, where $\omega_{1}(z)$ is the width of the Gaussian fit at a position equals to $f(z) / 2$. Thanks to this representation, it is also possible to define the apparent angle of the heap, $\alpha$, corresponding to the value of the slope when the inflection of the free surface is changed [8]. At each depth, this angle 
is calculated from $\tan (\alpha(z))=f(z) \exp ^{0.5} / \omega(z)$. For each granular media, the deflection and the apparent angle of the heap are non-constant during the filling step due to the position of the powder filling which is fixed during time (Fig. 3a). The drop height of the particles decreases with time and slows down their kinetic energy on the surface of the heap. This phenomenon is well described by Grasselli et al. [1].

For each granular media, the deflection fluctuates during the container filling by the granular medium. It can be considered as the sum of its mean value, $\bar{f}$, and its fluctuation, $\delta f(z)(f(z)=\bar{f}+\delta f(z))$. For couscous, the mean value of the deflection, or the mean value of the apparent angle of the heap (Table 2) and its fluctuation shape (Fig. 4) are almost the same. The fluctuation significantly increases in the bottom part of the cell, then more moderately in the middle before to rapidly increase again close to the top. For semolinas, the mean value of the deflection (or the mean value of the apparent angle of the heap) is higher than the one of couscous and it is all the more important as particles are smaller (Table 2). The deflection during pouring strongly fluctuates and probably in a periodic way (Fig. 4). While the heap deflection of the couscous bed increases during pouring, the one of the semolina bed increases then decreases alternatively (Fig. 4). Such a fluctuating behavior is reduced close to the top of the cell because of the progressive decrease of the particle drop height. Each particle family has therefore its own arrangement mode during the bed formation. As the mean deflection is smaller for couscous than semolinas (Table 2), then couscous are spread into a horizontal layer (heap of low amplitude) whereas the semolinas are distributed into sloping heap (as a 'dune'), constituted of oblique layers. We experimentally notice that the largest particles (couscous family) have a rebound ability much more important than the smallest (semolina family). Such effect leads to a heap formation with a deflection which increases monotonically between the bottom and the top of the cell. However, we observe that from a characteristic depth, $\lambda$, which is about the width of the cell (about $50 \mathrm{~mm}$ ), the behavior of both families is almost the same (Fig. 4). 


\subsection{Vertical force profiles in the center of the cell}

The both modes of the heap formation (layer mode for couscous or dune mode for semolinas) have an impact on the force distribution, $F(x=0, z)$, within the granular medium and measured in the center of the cell at different depths for the different selected wheat powders. Fluctuations of the force are not significant. Therefore its mean value, $F_{z}$, is sufficient to describe the mechanical state in the vertical direction. For each wheat powder, the local vertical force profile (Fig. 5) starts to be linear from the surface, then deviates from it (for semolinas this deviation is positive, i.e. $F_{z}$ becomes higher, and for couscous this deviation is negative, i.e. $F_{z}$ becomes lower) for decreasing until the bottom due to the the capacity of the bottom to redirect the vertical force laterally $[9,10,11,12]$.

Considering the first part from the bed surface, the vertical force profile is well fitted by a linear trend (Fig. 5), where the gradient, $S^{-1}$, is comprised between $53.10^{-4} \mathrm{~N} / \mathrm{mm}$ for fine semolina and $97.10^{-4} \mathrm{~N} / \mathrm{mm}$ for fine couscous (Table 3). These values are higher than the gradient of the equivalent hydrostatic state, $\phi \rho_{s} g \Omega$, defined as the weight of the granular column for which the section is equal to the surface of the probe $\Omega$ (Table 3 ). In fact, the gradient $S^{-1}$ can be associated with a 'pseudo'-hydrostatic weight (its value is approximatively equal to the hydrostatic value increased by 18\%) [5] taking into account all the contribution of forces acting on the probe and coming from the different parts of the force network $[2,3]$.

Globally, these vertical force profiles can be compared with Janssen's model $[4,10]$ which describes the normal stress at the bottom of an ensiled granular medium versus the height of the grain bed. This model introduces a characteristic length, the Janssen length $\lambda_{J}=D_{h} / 4 \mu K$ (where $D_{h}$ is the hydraulic diameter of the column, and $K$ is a coefficient which relates the horizontal redirection of the vertical stress), which allows to distinct a linear dependence of the pressure, consistent with a hydrostatic behavior (defined as the total weight of the granular bed which would weigh on the bottom) for heights lower than $\lambda_{J}$, and a constant 'saturation' pressure, induced by the walls which absorb the 
vertical force deflection for higher heights $[3,10]$. The local vertical profiles of couscous could be then assimilated to a like-Janssen behavior; the layer formation mode of the heap ensuring a hydrostatic mechanic state at each depth far from the bottom.

Table 3: Characteristics of the 'pseudo'-hydrostatic and the hydrostatic weight acting on the probe.

\begin{tabular}{lll}
\hline Name & $S^{-1}(\mathrm{~N} / \mathrm{mm})$ & $\phi \rho_{s} g \Omega(\mathrm{N} / \mathrm{mm})$ \\
\hline Fine semolina & $(53 \pm 3) \cdot 10^{-4}$ & $49 \cdot 10^{-4}$ \\
Semolina & $(65 \pm 2) \cdot 10^{-4}$ & $53 \cdot 10^{-4}$ \\
Fine couscous & $(97 \pm 3) \cdot 10^{-4}$ & $69 \cdot 10^{-4}$ \\
Medium couscous & $(76 \pm 1) \cdot 10^{-4}$ & $68 \cdot 10^{-4}$ \\
Large couscous & $(82 \pm 3) \cdot 10^{-4}$ & $69 \cdot 10^{-4}$ \\
\hline
\end{tabular}

\subsection{Local vertical force versus heap formation}

To compare the whole data coming from the free surface and the local vertical force, we defined a dimensionless parameter, $D$, which depends on the depth $z$ : $D(z)=\frac{f(z) \cdot S^{-1}}{F_{z}}$

This parameter highlights the correlation between the normalized force (by the 'pseudo'-hydrostatic state) measured at each depth in the center of the cell and the deflection of the heap which has contributed to the establishment of the local mechanical state. As for the deflection, we have written $D(z)$ as follow: $D(z)=\overline{D(z)}+\delta D(z)$, where $\bar{D}=\bar{f} \cdot S^{-1} / F_{z}$ and $\delta D=\delta f \cdot S^{-1} / F_{z}$. Excepted close to the bottom, where the interaction of the first poured particles leads to different organizations according to their dissipation properties (restitution coefficient and friction coefficient) [9], the behavior of the powders seems to follow a coherent distribution. Indeed, the dependence of $\bar{D}$ parameter with the depth indicates that the level of the local vertical force is correlated with the mean shape of the free surface (Fig. 6a). For couscous grains, the particle organization into a flat heap (small $f$ values), which can be assimilated to a 
layer distribution, is in agreement with the hypothesis of Janssen which deals with the lateral uniformity of the stresses [4]. This behavior, highlighted by a 'pseudo'-Janssen profile, is characterized by a lateral deflection of the force and therefore large values of $\delta D$. On the other hand for semolinas the particle organization into a sloping heap (large $f$ values) reduces the capacity of the lateral deflection inducing higher values of $\delta D$. For all particles the values of $\delta D(z)$, which integrate the fluctuations of the deflection and the normalized vertical force profile, seem to draw a master curve (Fig. 6b). $\delta D(z)$ does not almost vary from the bottom of the cell until the characteristic depth already highlighted and of the order of the width of the cell. Such an experimental result shows that during the powder pouring in the cell, the local force profile is relatively correlated with the fluctuations of the heap formation. Close to the surface of the cell, where the vertical force profile is 'pseudo'-hydrostatic, the fluctuations of the heap formation is mainly controlled by the lack of kinetic energy of the particle drop height.

\section{Conclusion}

This work deals with the experimental analysis of the impact of a granular medium ensiling history on the distribution of the local forces. For a 2D experimental configuration, the powder filling is characterized by the free surface evolution of the granular medium whereas the static intergranular contact network is represented by the vertical force measured in the center of the cell. The width of the cell, the filling conditions, and the particle properties (size and shape, coefficient of restitution, coefficient of friction), are the parameters which lead to define at least two different modes of heap formation: the horizontal layer mode and the oblique layer mode (dune). For all wheat-powders, the vertical force acting on a probe placed in the center of the cell is 'pseudo'hydrostatic until a characteristic depth of about the width of the cell. Bellow this depth, the lateral deflection of the force is correlated with the shape of the heap formed during the implementation of the column. The more the heap is 
sloping, the more the intensity of the local force is high with regard to its local 'pseudo'-hydrostatic state. Such a result allows considering more explicitly the history of the bed implementation on the force distribution within an ensiled granular medium.

\section{References}

[1] Y. Grasselli, H. Herrmann, G. Oron, S. Zapperi, Effect of impact energy on the shape of granular heaps, Granular Matter 2 (2) (2000) 97-100.

[2] L. Vanel, P. Claudin, J. Bouchaud, M. Cates, E. Clement, J. Wittmer, Stresses in silos: Comparison between theoretical models and new experiments, Physical Review Letters 84 (7) (2000) 1439-1442.

[3] L. Silbert, G. Grest, J. Landry, Statistics of the contact network in frictional and frictionless granular packings, Physical Review E $66(6,1)(2002)$.

[4] M. Sperl, Experiments on corn pressure in silo cells - translation and comment of Janssen's paper from 1895, Granular Matter 8 (2) (2006) 59-65.

[5] P. Richard, M. Nicodemi, R. Delannay, P. Ribiere, D. Bideau, Slow relaxation and compaction of granular systems, Nature Materials 4 (2) (2005) $121-128$.

[6] S. Mandato, B. Cuq, T. Ruiz, Experimental study of vertical stress profiles of a confined granular bed under static and dynamic conditions, European Physical Journal E 35 (7) (2012) .

[7] W. Beverloo, H. Leniger, J. Vandevelde, The flow of granular solids through orifices, Chemical Engineering Science 15 (3-4) (1961) 260-\&.

[8] Y. Grasselli, H. Herrmann, On the angles of dry granular heaps, Physica A $246(3-4)(1997) 301-312$.

[9] L. Vanel, D. Howell, D. Clark, R. Behringer, E. Clement, Memories in sand: Experimental tests of construction history on stress distributions under sandpiles, Physical Review E 60 (5, A) (1999) R5040-R5043. 
[10] G. Ovarlez, E. Clement, Elastic medium confined in a column versus the Janssen experiment, European Physical Journal E 16 (4) (2005) 421-438.

[11] S. Mandato, T. Ruiz, B. Cuq, What is Janssen's length doing in an agglomerator?, Powder Technology 238 (SI) (2013) 56-63.

[12] J. Zhu, Y. Liang, Y. Zhou, The effect of the particle aspect ratio on the pressure at the bottom of sandpiles, Powder Technology 234 (2013) 37-45. 

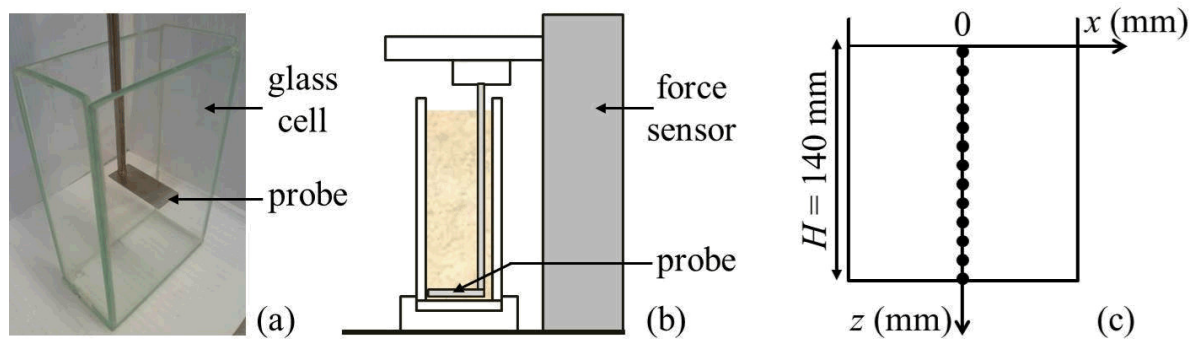

Figure 1: (a) Picture of the experimental glass cell and the probe, (b) schematic representation of the experimental set-up (side view), and (c) schematic representation of the probe positions in the cell (front view). 

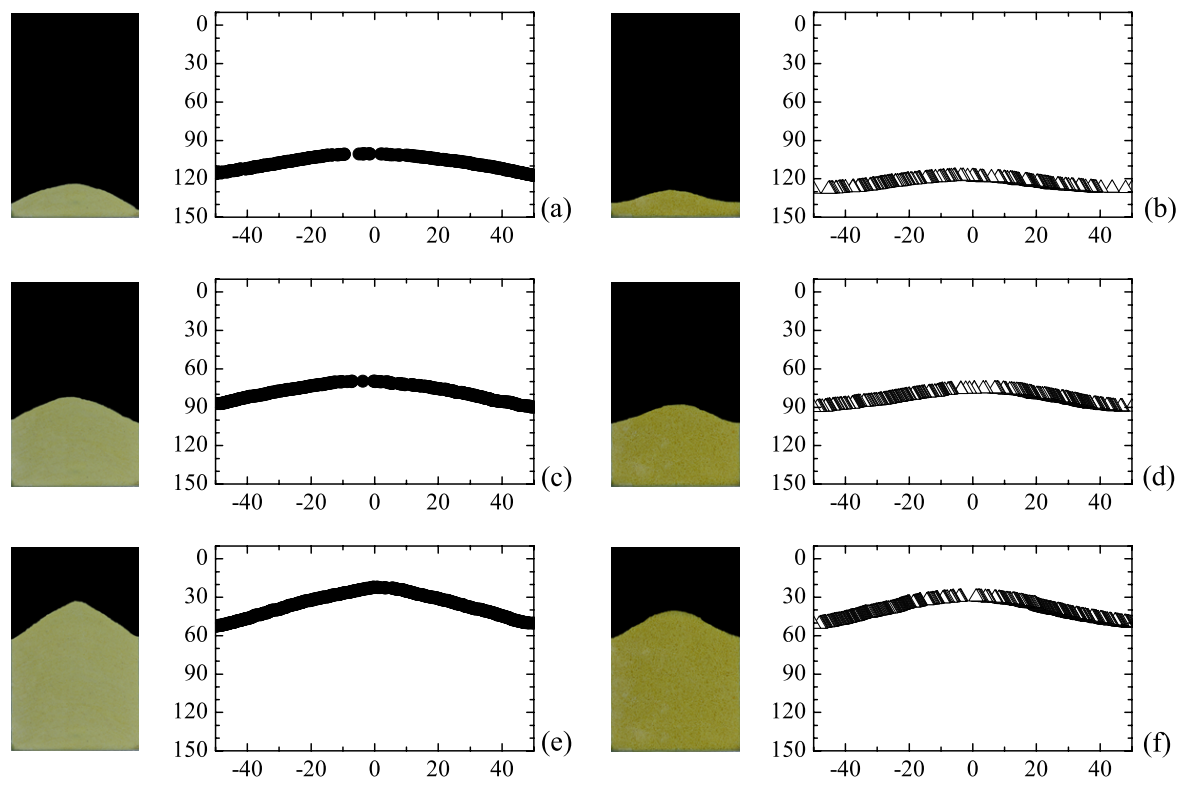

Figure 2: Pictures and captures the free surface of (a, c, e) semolina and (b, d, f) large couscous beds for a depth (at the wall) of about 20,70 and $120 \mathrm{~mm}$. 

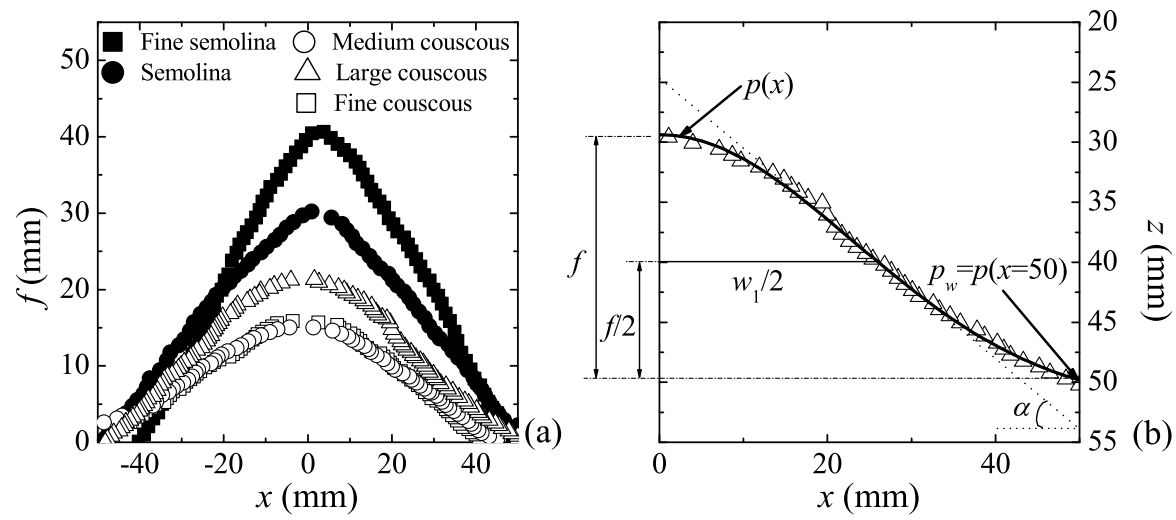

Figure 3: (a) Free surface at about $50 \mathrm{~mm}$ depth (at the wall) for all granular media, (b) half-free surface (scatter) and Gaussian fit of Eq. 1 (line) with fit parameters. 


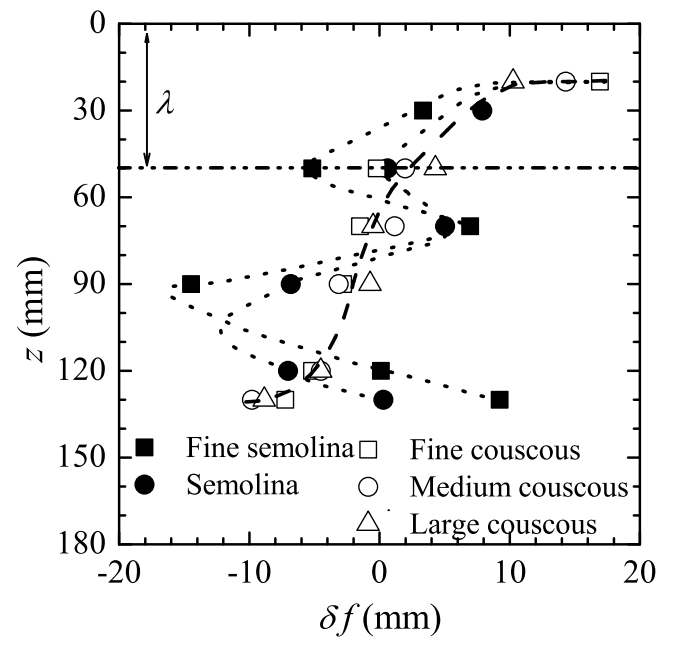

Figure 4: Fluctuation profile of the deflection heap for all granular media. The dashed and dotted lines represent guidelines. 

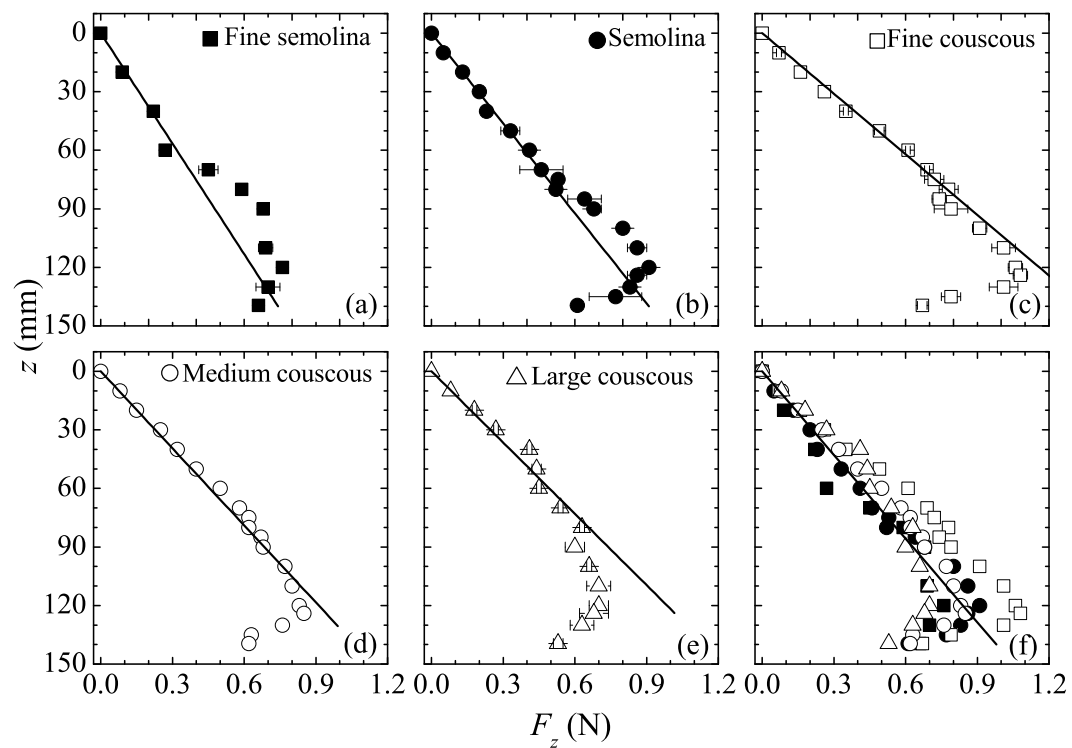

Figure 5: The vertical force for (a) fine semolina, (b) semolina, (c) fine couscous, (d) medium couscous, (e) large couscous, and (f) all wheat-powders as a function of the depth. The 'pseudo'-hydrostatic profile is represented by a line. 

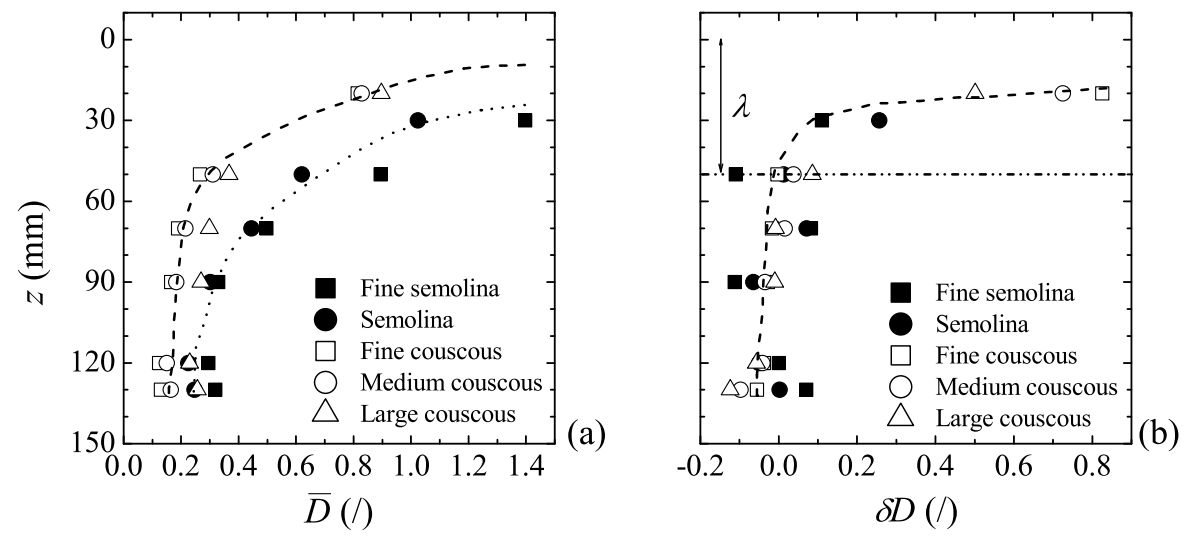

Figure 6: Profiles of the dimensionless parameter for all granular media: (a) mean value and (b) fluctuation. The dashed and dotted lines represent guidelines. 